\title{
Clinical and functional evaluation of a gaze controlled system for the severely handicapped
}

\author{
P Thoumie ${ }^{1}$, JR Charlier ${ }^{2}$, M Alecki ${ }^{3}$, D D'Erceville ${ }^{4}$, A Heurtin ${ }^{5}$, JF Mathe ${ }^{5}$, G Nadeau ${ }^{4}$ and L Wiart $^{3}$ \\ ${ }^{1}$ Service de Rééducation Fonctionnelle, Groupe hospitalier Bichat Claude Bernard, 75018 Paris; ${ }^{2}$ Equipe Vision et \\ Regard, Institut de Technologie Médicale, CHU de Lille, 59037 Lille; ${ }^{3}$ Centre de Rééducation Fonctionnelle La Tour \\ de Gassies, 33523 Bruges; ${ }^{4}$ Centre de Rééducation Fonctionnelle de l'Arche, 72650 Saint Saturnin, ${ }^{5}$ Service de \\ Rééducation Fonctionnelle, Hopital Saint Jacques, CHU de Nantes, 44000 Nantes, France
}

\begin{abstract}
This preliminary study evaluates the suitability of a gaze controlled communication system for severely handicapped patients. The system drives a computer by movements of the patient's pupils. The capacity to communicate by producing text on a screen was assessed in 30 patients, 22 traumatic tetraplegics and 8 patients whose handicaps were due to other causes. The rate at which they learned to use the system, and the speed of their word processing were measured. Those tetraplegics who had used other communication systems found the gaze controlled system tiring, and its lack of other peripherals limiting. But its true application seems to be as the sole available interface for the most severely handicapped who can neither move nor speak. Peripherals and improved calibration systems are presently being developed.
\end{abstract}

Keywords: tetraplegia; communication system; gaze

\section{Introduction}

The developments in emergency medicine that have occurred over the past few decades have meant that the care given to severely injured patients has improved dramatically, ${ }^{1,2}$ but much remains to be done to make the subsequent quality of life for these patients satisfactory. As a consequence, the most severely handicapped patients are totally dependent on their family or some other person for their daily needs. The most severely disabled may not only be suffering from lack of mobility, they may also be unable to speak, and hence have lost the ability to communicate. This physical isolation is made worse by a loss of relations with others, leading to a situation that has been called the great internal monologue.

Advances in technology over the past 20 years or so have provided the severely handicapped with systems for controlling their environment. A variety of techniques allow them some degree of independence in managing their daily lives. ${ }^{3-5}$ Computers are a fundamental component of rehabilitation systems for those who can no longer write. More recently, control systems using vocal or head movement signals have been used to run the home environment.

For persons with reduced functions of hands and language, the use of gaze command appears to be of great potential interest. Several instruments have

Correspondence: Dr P Thoumie already been proposed for this purpose. ${ }^{6-8}$ However, no thorough clinical evaluation of these instruments has been performed until now. The present multi-center study was carried out to evaluate a new gaze controlled system in three rehabilitation centers. The goal was to assess the acceptability, reliability and reproducibility of the system when used by the most severely handicapped patients.

\section{Equipment and methods}

\section{Equipment}

The gaze controlled system includes an eye movement sensor, a designation board and a microcomputer (Figure 1). The eye movement sensor is based upon the Hirschberg principle ${ }^{9}$ and determines gaze orientation from the relative position of two images: the reflexion of a light source over the cornea and the pupil. This technique allows determinations of gaze direction which are absolute, independent from head and reproducible over time. ${ }^{10}$ It is less invasive and much more precise (about one degree of visual angle) than some of the techniques used in previous reports. For example, the electro-oculography technique ${ }^{6,8}$ requires skin electrodes to record biopotentials. Its accuracy is limited by drifts of the electrode potentials and various sources of artefact. Greater accuracy allows the selection of a higher number of triggering actions and does significantly improve the efficiency of 


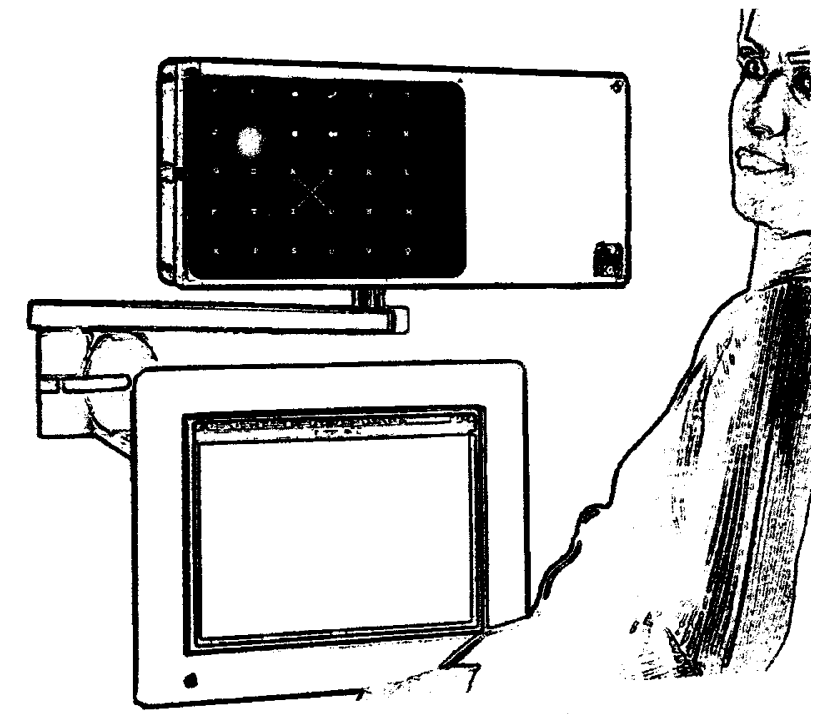

Figure 1 General set-up of the gaze controlled system showing the designation board (top) and the monitor of the microcomputer (bottom). The eye movement sensor views the eyes from a small aperture at the centre of the designation board

the device. That means that one letter of the alphabet can be selected with only one gaze fixation. If the accuracy is lower (for example four designation areas with the electro-oculographic technique) selecting one character of the alphabet requires several operations, which is more time consuming and also requires more attention from the patient.

In the gaze controlled system, measurements of gaze direction are performed on the image from a near infra-red $(880 \mathrm{~nm})$ solid state camera. The automated analysis of the video signal is performed at a rate of 30 $\mathrm{Hz}$ by specific electronics and a microprocessor. Automated pattern recognition algorithms ${ }^{11,12}$ provide measurements which are free from artefacts encountered in a large number of subjects such as partial masking of the pupil by eye lashes or eye lids and parasitic reflections.

An ultrasound sensor is used to measure the distance to the head and control the focus of the image sensor. Two micro-stepping motors control the angular position of two mirrors defining the orientation of the eye movement sensor. Once the image of the eye is detected over the sensor, this device automatically tracks the eye to compensate for head movements. When the image of the eye is lost, it automatically scans over an area of $10 \times 10 \mathrm{~cm}$ to search for the eye, allowing a relative freedom of head movements. The designation board includes a $5 \times 6$ matrix of designation area, each subtending a visual angle of $4 \times 4$ degrees (Figure 2) and comprising letters of the alphabet and control keys (space, line advance, erase and print) The codes of the designated areas are sent to the microcomputer via the keyboard input.

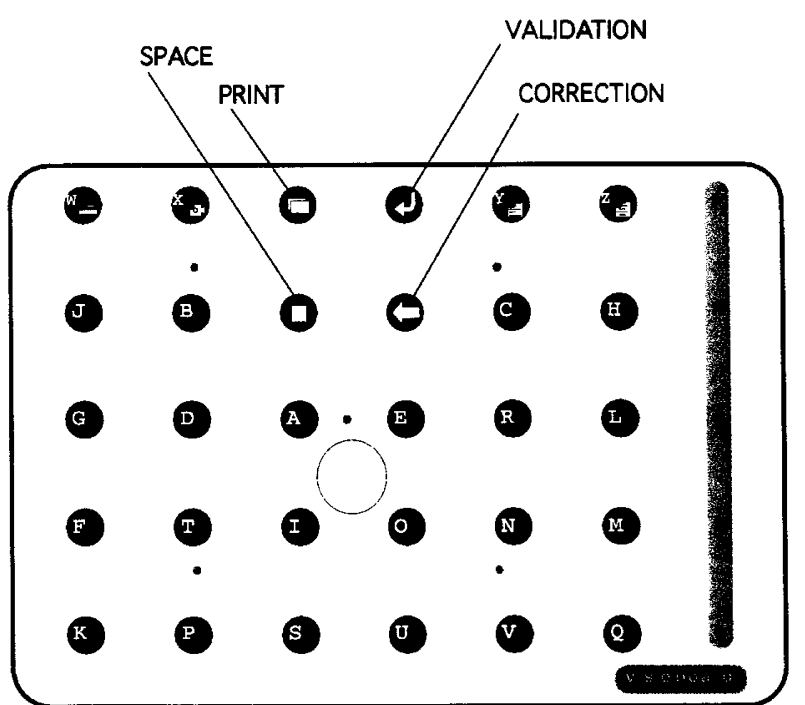

Figure 2 The designation board showing the central window behind which the eye movement sensor is located and a matrix of $5 \times 6$ designation areas including the letters of the alphabet and four specific commands: -1- for writing a space, -2- for printing the text, -3- for erasing the last character -4for advancing one line in the text

\section{Operating principle}

Once the head is detected by the ultrasound sensor, the device automatically searches for the eye until it is detected within the viewing field of the camera. The next step is a calibration of the sensor which involves reference measurements obtained while the subject is fixating five light sources located at different locations within the designation board. Thereafter, the device can be used simply be looking at the designation areas. Validation of designations is based on the duration of fixations. In the present experiments, the threshold for minimum duration of fixation was set at 300 milliseconds (msec) in order to allow for free scanning of the designation board by the subjects who were not familiar with the specific arrangement of letters. (letters which were more frequent in the French alphabet were located in the center of the board). Visual feedback is provided to the user by illuminating the designated area for $300 \mathrm{msec}$ after validation.

\section{Patients}

A total of 30 patients (25 men, five women) were recruited at the three evaluation sites and tested between 10 October 1995 and 1 May 1996. The details for each patient are shown in Table 1. Their average age was 31 years and they had been handicapped for an average of 4 years (range: 3 months-27 years). Most of them had cervical medullary lesions which had resulted in total tetraplegia. The other patients had varying degrees of handicap, and each was included in the protocol to 
Table 1 General data of the 30 patients included in the study

\begin{tabular}{|c|c|c|c|c|c|}
\hline Patient & Pathology & Sex & Age & Duration & Success \\
\hline 1 & Tetraplegia C4 & M & 50 & 2 years & - \\
\hline 2 & Tetraplegia C4 & $\mathrm{M}$ & 33 & 1 year & + \\
\hline 3 & Tetraplegia C4 & $\mathrm{M}$ & 18 & 6 months & + \\
\hline 4 & Tetraplegia C5 & M & 29 & 7 months & - \\
\hline 5 & Tetraplegia C5 & $\mathrm{F}$ & 66 & 1 year & - \\
\hline 6 & Tetraplegia C5 & M & 44 & 6 months & + \\
\hline 7 & Tetraplegia C5 & M & 30 & 5 years & + \\
\hline 8 & Tetraplegia C5 & M & 17 & 4 months & + \\
\hline 9 & Tetraplegia C6 & M & 35 & 7 months & - \\
\hline 10 & Tetraplegia C6 & M & 20 & 3 months & - \\
\hline 11 & Tetraplegia C6 & M & 17 & 3 months & + \\
\hline 12 & Tetraplegia C6 & M & 21 & 7 months & + \\
\hline 13 & Tetraplegia C6 & M & 18 & 7 months & + \\
\hline 14 & Tetraplegia C6 & M & 25 & 1 year & + \\
\hline 15 & Tetraplegia C7 & M & 19 & 5 years & - \\
\hline 16 & Tetraplegia C7 & M & 18 & 6 months & + \\
\hline 17 & Tetraplegia C7 & M & 44 & 11 months & + \\
\hline 18 & Tetraplegia C7 & M & 27 & 13 years & + \\
\hline 19 & Tetraplegia C7 & M & 26 & 4 months & + \\
\hline 20 & Tetraplegia C7 & M & 24 & 6 months & + \\
\hline 21 & Tetraplegia C7 & M & 26 & 1 year & + \\
\hline 22 & Tetraplegia C7-C8 & $\mathrm{F}$ & 51 & 6 months & - \\
\hline 23 & Locked -In Syndrome & M & 48 & 5 years & - \\
\hline 24 & Locked -In Syndrome & $\mathrm{M}$ & 40 & 3 years & + \\
\hline 25 & Spinal Amyotrophy & M & 29 & 20 years & + \\
\hline 26 & Spinal Amyotrophy & M & 30 & 27 years & + \\
\hline 27 & $\begin{array}{l}\text { Amyotrophic Lateral } \\
\text { Sclerosis }\end{array}$ & $\mathrm{F}$ & 53 & 11 years & + \\
\hline 28 & $\begin{array}{l}\text { Cerebral } \\
\text { Palsy }\end{array}$ & $\mathrm{F}$ & 27 & 27 years & + \\
\hline 29 & Hemiplegia & M & 19 & 2 years & + \\
\hline 30 & $\begin{array}{l}\text { Brachial Plexus } \\
\text { Injury }\end{array}$ & M & 20 & 8 months & + \\
\hline
\end{tabular}

evaluate the usefulness of the system in a wide range of situations.

\section{Experimental protocol}

Successful use of the equipment was defined as the ability to produce a sentence on the computer screen that contained at least one line of characters freely selected by the patient. A clinical file was kept on each patient. The file contained the following evaluation parameters: (i) Clinical information on the injuries, motor loss and date of inset; (ii) A record of the learning session showing the number and duration of sessions, plus the success or failure in using the equipment; (iii) A record of the reproducibility of use and the reports of the patient, the care team and the project supervisor on the use of the equipment; (iv) Evaluation of the fatigue resulting from use of the gaze controlled system; (v) An overall assessment of the equipment by comparison with other systems of communication previously used by the patient.

Each group was required to submit a report so as to follow assessment of the interest shown in the equipment, the opportunities for its use for the disabilities concerned, future development paths or other types of patients to be tested.

\section{Results \\ Overall results \\ Of the 30 patients included in the trial, 22 learned to produce a sentence of 50 characters at least once.}

\section{Failures}

The eight patients who did not succeed in using the gaze controlled system included seven tetraplegics [level C4 (one case), C5 (two cases), C6 (two cases), C7 (two cases) and $\mathrm{C} 8$ (one case)], and one patient having locked-in syndrome. Failure was declared after an average of four sessions (range: 1-9). The main cause of failure was inability to calibrate the eye (six cases); the others were an inability to correctly position the patient's head (one case) and psychological rejection (one case).

\section{Successes}

The 22 patients who succeeded in using the gaze controlled system at least once are listed in Table 1. The number of sessions required varied from just one (17 cases) to two sessions (three cases), five sessions (one case) and 18 sessions (one case). The patients who succeeded in using the system on the first attempt used the system in an average of seven sessions (range 131). Twenty-three subjects reported feeling tired; they specifically mentioned the neck muscles (12 cases) or eyes (one case), with a prickling sensation or eye muscle fatigue and finally back pain. No one reported a headache. The fatigue appeared on average after 20 $\pm 10 \mathrm{~min}$ (range: $10-40 \mathrm{~min}$ ).

The reproducibility among the 18 patients who successfully completed more than one trial was considered good in 12 cases, average in two cases and poor in four cases. Twelve patients considered the system acceptable and four considered it to be not acceptable. Of the 16 patients who responded, nine considered the system to be convenient, six considered it slow, and one thought it unusable. The testing team (15 responses) considered the apparatus to be simple (12 replies), complex (two replies) and unusable (one reply). Lastly, the judgment of the local project leader on 16 cases was that the apparatus was suitable (eight cases), difficult (six cases), and unsuitable (two cases).

The patients required $1 \mathrm{~min} 30 \mathrm{~s}$ to $30 \mathrm{~min}$ (median $6 \mathrm{~min}$ ) to write 50 characters. Comparison with other equipment showed that they were all preferred to the gaze controlled system. Three patients had been using a controlled system that used head movements for several weeks or months. One found this head controlled system better, one preferred the gaze control, and one considered them equivalent. 


\section{Discussion}

\section{Problem summary}

This study was carried out to assess the feasibility of using the gaze controlled system as an interface for severely handicapped patients. We evaluated their ability to use the system to write a sentence of 50 characters on a monitor screen.

The evaluation was done at only three centers because few of the gaze controlled systems are presently available, teams had to be trained, but the results had to be reliable and obtained quickly. Hence, the patients recruited were somewhat similar, which is a positive point for those with medullary damage, but it restricted use of the system by other potential groups. It should also be remembered that the apparatus is still in the prototype stage. This made it difficult to evaluate and compare the system to other systems, most of which have peripheral applications. The gaze controlled system is still limited to word processing using a simple, but uninspiring system. A clinical study was therefore needed to assess the system and identify any future developments indicated by the patients and the healthcare team.

\section{Patient selection}

Most (22/30) of the patient who took part in this study had cervical medullary damage. They were selected because the tetraplegic population is one of the greatest users of technological support and communication systems. These patients are young, with a long life expectancy, have no higher function problems, and their need for outside help cannot always be satisfied. Most of these patients have not been handicapped for long, but this does not alter their capacity to use the equipment. However, as many of them have not completely come to terms with their situation, they may have little or no motivation to continue the experiment beyond the initial stages. Medically, those with tetraplegia have not only lost the use of their arms, which requires a prosthesis like the gaze controlled system, but degenerative disorders may also affect the pupils (myosis or sympathetic paralysis, or dilatation during an attack of autonomic hyperreflexia). This evaluation allows the assessment of the way these degenerative disorders interfere with the use of the gaze controlled system. This trial was primarily a feasibility test for those patients suffering from other disorders. There were too few of them, in some instances, for the findings to be extrapolated to a larger population.

\section{Failures and limiting factors}

The main cause of failure with the gaze controlled system was an inability to calibrate the system; this occurred for eight of the subjects, seven of whom had tetraplegia (lesions at $\mathrm{C} 4-\mathrm{C} 8$ ). There are several possible reasons for this, such as myosis due to the medullary lesion, stress caused by the experiment itself, or environmental problems, particulary lighting. A lighting problem was noted on several occasions, but it cannot be the cause of repeated failure as it can be altered by trying other situations (shading the room, altering the lighting and the position of the ocular keyboard, orientation of the keyboard, or even moving to a new trial site).

In addition to failures due to lack of calibration, other factors limiting the use of the gaze controlled system came to light during the trials. The main one was fatigue, principally of the neck muscles, or a psychological fatigue that usually caused the patient to stop the trial after $20-30 \mathrm{~min}$, regardless of the results they were getting. The muscular component is directly linked to the fact that most of patients did not want to use a head rest.

The psychological problems occurred in several patients. In the short term they led to rejection of the experiment because of the image of the handicapped they sent to the team, particularly for patients who had not completely come to terms with their loss of communication functions. ${ }^{13}$

Like other mechanical aids used to control the paraplegic patient's environment, the psychological responses of the patients to the use of the equipment varied. One of the clearest responses during the trial was the notion that the gaze controlled system could make the handicapped person look even more disabled in the eyes of those around him (family, other patients). This poses the problem of the balance between a functional advantage and a psychological disadvantage.

One of the features which contributed to the poor psychological tolerance of the system was the constant need for someone to start the machine, set the patient in position and do the calibration. This dependence, at least during the initial setting up of the apparatus, is linked to the ergonomics of the optical keyboard. The keyboard currently used is placed $30-40 \mathrm{~cm}$ from the eyes, which limits the visual field. This is important for system tolerance by patients who want to have a break from using the gaze controlled system after 20-30 min, as it does not really allow them to manage their immediate environment.

\section{The gaze controlled system and other systems of} communication

Most of the patients who took part in the trials, particularly the tetraplegics, had previously used one or more environment control and/or communication systems, so that they were able to compare the gaze controlled system with them. The resulting evaluation was that, provided the patient could use a keyboard, or a single-lever device (eg, wrist control), or voice control, all these systems were preferred to the gaze controlled system, either because of the ergonomics or because of the better performance. 
An alternative to the existing system was considered by those patients with the greatest handicap (tetraplegic individuals with a respiration difficulty which prevented communication, locked-in syndrome patients). The gaze controlled system is a real alternative for these patients, particularly because there is no direct link between the patient and the system, such as there is for the Head-master controlled system. But this was tempered by the fact that the other systems were much more functional, because the current gaze controlled system has no peripherals.

The most adept patients were able to produce a sentence of 50 characters in less than 2 min but some other patients who adapted less quickly took 20-30 min. This was generally due to unstable recognition of the ocular movements and fatigue due to the abnormal movements required.

\section{Future prospects}

The present study provides useful data on patients with medullary injuries and shows that the system is feasible, but is limited by its lack of reproducibility and capacity for repeated use, compared to other currently available systems, and its tolerance in use. Some other patients with other types of injuries were tested, but several pieces of useful information have emerged. The patients for whom the gaze controlled system will be most useful are those who have no alternative means of communication, particularly those with total loss of movement of all four limbs and the power of speech. This can occur after damage to the central nervous system (locked-in syndrome), or degenerative disorder, such as lateral amyotrophic sclerosis. It might also be of transient use for those with neurological disorders producing this type of loss, such as Guillain - Barré syndrome and tetraplegic patients during the recovery phase. These patients never communicate, and do not speak most of the time except by a system of eye movements based on yes/no responses. Even in its limited configuration as a word processor, this gaze controlled system may be of use to patients in this extreme condition, as there is no other suitable technology available. But the gaze controlled system does not seem to be able to compete with existing system outside of these extreme situations, unless certain features are improved. The main problem seems to be the reliability of the system, which is due to difficulties of calibration and reproducibility. Only two out of three patients with medullary injuries $(22 / 30)$ seem to be potential users because of calibration problems. These problems can be identified in the first session (17 patients who successfully used an apparatus were calibrated in the first session). But even those initially successful patients ran into problems of system reproducibility. It was considered to be good by $2 / 3$ subjects who succeeded in the first trial, but only by $1 / 2$ patients overall.

The second problem is fatigue, either of the neck muscles or of the eyes. This suggests that the system in its present form is not suitable for prolonged use, or for use outside the home.

The fact that the patient cannot set up the machine by himself also reflects a fundamental problem with all systems of communication and environmental control. The gaze controlled system, like other current systems, requires another person for installation and start-up. Improved techniques have been developed recently for the storage and reuse of calibration data and a system that recognizes a look from the patient once he/she is within the camera field.

The last problem is clearly the driving of peripherals: all the existing systems are interfaces that allow the use of systems to control the environment or multimedia systems. Any true comparison with existing systems will depend on these improvements.

\section{Conclusion}

The gaze controlled system is a novel technological response to the need for communication by the severely handicapped. The system differs from other existing systems in that there is absolutely no physical contact between the patient and the machine. This preliminary study has revealed the limiting factors to be calibration problems (specific to patients with medullary injuries), fatigue during use of the equipment, and poor performance in terms of speed of word processing.

Nevertheless, the system is unmatched in its suitability as a communication tool for the most severely handicapped, for whom there is presently no other suitable system. The very existence of this equipment makes the shortcomings listed above of secondary importance for these patients. The future possibilities of this gaze controlled system should be confirmed by clinical studies in which they are assessed by the most severely handicapped (patients with high-level tetraplegia, locked-in syndrome, lateral amyotrophic sclerosis, or polyradiculoneuritis). The gaze controlled system has not been improved beyond the word processing stage, and could be suitable for re-evaluation by patients with medullary damage, or even less severe handicaps, such as sufferers from myopathy or IMC, for whom the equipment may be suitable.

\section{Acknowledgements}

This work was supported by a grant from DELTA-7 association.

\section{References}

1 Carter E. Experiences with high tetraplegics. Paraplegia 17: 1979; $140-146$.

2 Ravichadran G, Silver JR. Survival following traumatic tetraplegia. Paraplegia 20: 1982; 264-269.

3 Parish JG. A study of the use of electronic environmental control systems by severely paralysed patients. Paraplegia 17: 1979; $147-152$. 
4 Kwee $\mathrm{HH}$ et al. First experimentation of the spartacus telethesis in a clinical environment. Paraplegia 21: 1983; 275-286.

5 Van Laere M, Duyvejonck R. Environmental control and social integration of a high-lesion tetraplegic patient: case report. Paraplegia 1986; 24: $322-325$.

6 Kate JH, Van der Meer P. An electro-ocular switch for communication of the speechless. Med. Prog. through Technology 10: $1984 ; 135-140$

7 Frey LA, White KP, Hutchinson TE. Eye-gaze word processing. IEEE Trans Systems, Man Cybernetics 1990; 20,4: 944-950.

8 Kaczmarek R. Commande oculaire pour l'aide à la communication et au contrôle de l'environnement par l'handicapé moteur. Motricité cérébrale 1992; 13: 24-30.

9 Hirschberg J. Uber die Messung des Schieldgrades und die Dosierung der Schieldoperation. Zentral Prakt Augenheild 1885; 8: 325 .

10 Buquet C, Charlier JR. Quantitative assessment of the static properties of the oculomotor system with the photo-oculographic technique. Med.\& Biol. Eng. and Comput. 1994; 32: 197 204
11 Charlier JR, Bariseau JL, Paris V. Dispositifs de traitement du signal pour l'analyse d'images de l'oeil Brevet France N.85.139.11. Extension aux pays européens, 1985.

12 Charlier JR. et al. Real term pattern recognition and feature analysis from video signals applied to eye movement and pupillary reflex monitoring. Doc. Ophtalmol. Proc. Series 1985; 42: $181-189$.

13 Judd FK, Brown DJ. Psychiatry in the spinal injuries unit. Paraplegia 1987; 25: $254-257$

\section{Appendix}

The three gaze controlled systems used in this study were experimental devices built by the Metrovision Company (Villeneuve d'Ascq, France) under J Charlier specification. This device will be made available in the near future, after several technical improvements which have been suggested by the present study. 